\title{
Miositis osificante traumática en recién nacido: reporte de caso
}

\section{Traumatic Myositis Ossificans in Newborn: A Case Report}

\author{
Daniel López H. ${ }^{1}$ Carmen Labbé C. ${ }^{2}$ Marcelino Suazo R. ${ }^{2}$ Nicolás Toledo A. ${ }^{2}$
}

${ }^{1}$ Hospital San Juan de Dios Curicó, Curicó, Chile

Dirección para correspondencia Daniel López H, MD, Madrid 555,

2 Facultad de Ciencias Médicas, Universidad de Talca de Chile, Curicó, dpto. 802, Curicó, Chile (e-mail: danlophurtado@gmail.com). Chile

Rev Chil Ortop Traumatol 2021;62(3):e232-e236.

\section{Resumen \\ Palabras Clave \\ - miositis osificante \\ - neonatología \\ - ortopedia \\ - prematuridad \\ - recién nacido \\ - traumatismo}

La miositis osificante traumática (MOT) es una enfermedad en la que ocurre osificación heterotópica en dos a cuatro semanas tras uno o múltiples traumatismos. El objetivo de este artículo es describir las características clínicas y radiológicas de un caso de MOT en un recién nacido ( $\mathrm{RN}$ ) después de la canulación intravenosa de vía periférica, poco frecuente en la práctica clínica en neonatología. Presentamos a un RN pretérmino de 33 semanas en que, a los 20 días de vida, se evidenció lesión tumoral en el tercio distal del antebrazo izquierdo de $3 \mathrm{~cm}$ por $2 \mathrm{~cm}$, que no impresionaba dolor, ni limitación a la movilización, y en la que no había signos infecciosos. El resto del examen físico osteomuscular era normal. En la zona de lesión, tres semanas antes, se había instalado un catéter intravenoso periférico (CIVP). Una radiografía del antebrazo izquierdo demostró lesión calcificada al nivel de las partes blandas, sin disrupción de las estructuras óseas adyacentes; la ecografía del antebrazo reveló una imagen focal ovalada, de contornos parcialmente definidos, con sombra acústica posterior; el resto de los estudios de huesos largos era normal. Los niveles séricos de fosfatasa alcalina, calcio, fósforo también eran normales. En vista de la lesión tumoral al examen físico y la imagen calcificada en partes blandas a través de radiografía simple, con antecedente de microtraumas de VVP, se concluyó MOT. Se hizo seguimiento, con disminución del tamaño hasta que la lesión desapareció a los cuatro meses. No requirió control radiológico. La MOT es infrecuente en el RN, y, en general, la resolución es autolimitada y tiene buen pronóstico.

Nivel de evidencia IV recibido

08 de agosto de 2019

aceptado

06 de agosto de 2021
DOI https://doi.org/

$10.1055 / \mathrm{s}-0041-1739538$ ISSN $0716-4548$. (c) 2021. Sociedad Chilena de Ortopedia y Traumatologia. All rights reserved.

This is an open access article published by Thieme under the terms of the Creative Commons Attribution-NonDerivative-NonCommercial-License, permitting copying and reproduction so long as the original work is given appropriate credit. Contents may not be used for commercial purposes, or adapted, remixed, transformed or built upon. (https://creativecommons.org/ licenses/by-nc-nd/4.0/)

Thieme Revinter Publicações Ltda., Rua do Matoso 170, Rio de Janeiro, RJ, CEP 20270-135, Brazil 


\section{Abstract}

\section{Keywords \\ - myositis ossificans \\ - neonatology \\ - orthopedics \\ - prematurity \\ - newborn \\ - trauma}

Traumatic myositis ossificans (TMO) is a disorder in which heterotopic ossification occurs two to four weeks after one or multiple traumas. The goal of the present article is to describe the clinical and radiological characteristics of a case of TMO in a newborn (NB) after a peripheral intravenous cannulation, a rare procedure in the clinical practice of neonatology. The patient is a premature 33-week-old NB who, 20 days after birth, presented with a $3 \mathrm{~cm} \times 2 \mathrm{~cm}$ lump in the distal third of the left forearm that did not seem to cause pain or to limit movements, and with no evidence of infection. The rest of the physical exam was within normal limits. Three weeks before the lesion, a peripheral intravenous catheter (PIVC) was placed in that area. A radiograph of the left forearm showed soft-tissue calcification without disruption of adjacent bone structures. Ultrasound revealed a focal, oval soft tissue lesion with partially-defined borders and posterior acoustic shadow; the rest of study showed normal long bones. The serum levels of alkaline phosphatase, calcium, and phosphorus were all normal. In view of the tumor lesion on the physical examination and the calcified image in softtissue on plain X-ray and a recent history of PIVC microtrauma, we reached to the diagnoses of TMO. During the follow-up, the lesion decreased in size until it completely disappeared four months after the diagnosis. No radiological control was needed. Uncommon in NBs, TMO is generally self-limited and with a good prognosis.

\section{Introducción}

La formación de hueso laminar no neoplásico en los tejidos blandos, donde normalmente no existe, se denomina miositis osificante (MO). Sin embargo, sería más preciso describir como miositis osificante la afectación de los músculos esqueléticos $\mathrm{y}$, como osificación heterotópica $(\mathrm{OH})$, la afectación de los tejidos blandos en general. ${ }^{1}$ Samuelson y Coleman ${ }^{2}$ dividen la MO en cuatro grupos distintos: 1) miositis osificante progresiva o fibrodisplasia de herencia autosómica dominante; 2) miositis osificante asociada a enfermedad crónica; 3) miositis osificante traumática (MOT); y 4) miositis osificante pseudomaligna (no traumática).

La MOT es, quizás, la forma más común, y representa entre el $60 \%$ y el $75 \%$ de los casos. ${ }^{3}$ En un estudio retrospectivo publicado en el 2017, Sferopoulos et al., ${ }^{1}$ analizaron a 22 pacientes ( 11 niñas y 11 niños), con edades entre 3 y 14 años en el momento del diagnóstico, de los cuales 18 (81\%) casos correspondían a MOT. La enfermedad fue descrita por primera vez por Reidel en 1883, seguido por Dejerne y Ceiller, quienes la observaron en soldados que sufrieron lesiones en la médula espinal durante la Primera Guerra Mundial. ${ }^{4}$ Los datos epidemiológicos resaltan una prevalencia en hombres entre las edades de 30 a 40 años, siendo los deportistas la población de mayor riesgo. ${ }^{5}$ La lesión usualmente ocurre en los grandes grupos musculares del muslo (80\% de los casos) y la extremidad superior de los hombres jóvenes. ${ }^{1,6}$ La patogenia actualmente no es del todo clara, pero se ha postulado la participación de las proteínas morfogenéticas óseas (PMOs), que estimulan las células madre del huso mesenquimatoso, que luego migran al área lesionada y se transforman en fibroblastos y, finalmente, en osteoblastos. ${ }^{7}$ Clínicamente, es un tumor, que puede o no mostrar signos de inflamación local, precedidos por un trauma. ${ }^{6}$

El objetivo de este manuscrito es describir las características clínicas y radiológicas de un caso de MOT en un recién nacido (RN) después de la colocación de un catéter intravenoso periférico (CIVP), poco frecuente en la práctica clínica en neonatología.

\section{Caso clínico}

Un RN pretérmino de 33 semanas, del sexo femenino, producto de embarazo controlado, con diagnóstico prenatal de gastrosquisis, rotura prematura de membranas de 22 horas de evolución, obtenida por cesárea de urgencia, con peso al nacer de $1.900 \mathrm{~g}$, talla al nacer de $38 \mathrm{~cm}$, puntuación de 6 en la prueba de APGAR al minuto y de 8 a los 5 minutos. Se realizó cirugía abdominal de corrección del defecto de pared, sin complicaciones.

A los 20 días de vida, se evidenció una lesión tumoral en el tercio distal anteroposterior del antebrazo izquierdo, de $3 \mathrm{~cm}$ por $2 \mathrm{~cm}$, indurada, que no impresionaba dolor, ni limitación a la movilización, y sin signos infecciosos. Hubo instalación de un CIVP en la zona de lesión descrita en la primera semana de vida, y el RN no recibió gluconato de calcio por este CIVP. No hubo hallazgos de dismorfias en el resto del examen físico.

A los 22 días de edad, se solicitó radiografía ( $\mathrm{Rx})$ del antebrazo izquierdo (-Figura 1), que mostró una lesión calcificada al nivel de las partes blandas, en región dorsal, de aspecto radial, sin disrupción de las estructuras óseas adyacentes. La ecografía del antebrazo izquierdo (-Figura 2) reveló una imagen focal ovalada, de contornos parcialmente definidos, con sombra acústica posterior, que no presentaba flujo al Doppler. El resto de estudios radiológicos de huesos 


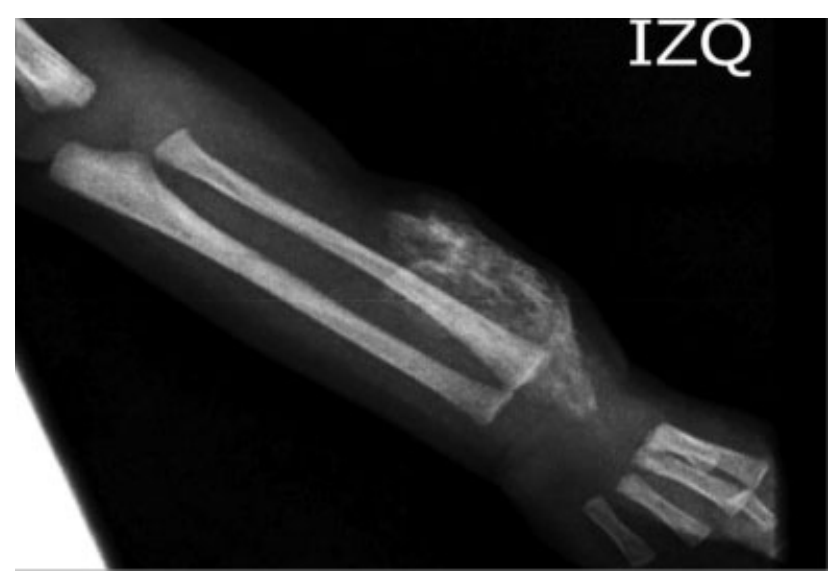

Fig. 1 Radiografía del antebrazo izquierdo de recién nacido: lesión calcificada al nivel de las partes blandas, en región dorsal, de aspecto radial, de predominio en la periferia, y con zona central parcialmente radiolúcida. Entre la lesión y el hueso subyacente, hay área radiolucente. No impresiona disrupción de las estructuras óseas adyacentes.

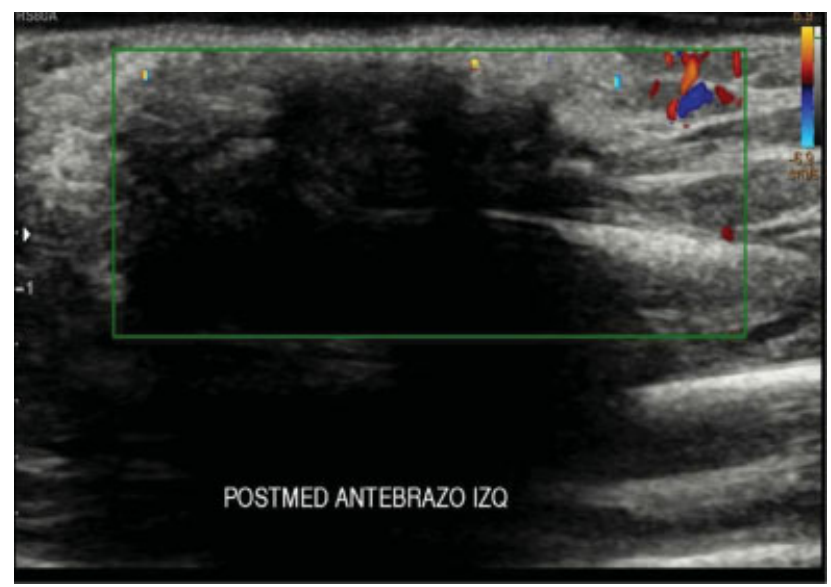

Fig. 2 Ecografía del antebrazo izquierdo: imagen focal ovalada, de contornos parcialmente definidos, con sombra acústica posterior, que no capta flujo al Doppler. Abreviatura: POSTMED, postmedicación.

largos era normal. El hemograma, la velocidad de sedimentación globular (VSG), y los niveles de proteína C reactiva, calcio, fósforo, fosfatasa alcalina (FA), lactato deshidrogenasa (LDH), y creatina quinasa (CQ) eran normales. Ante estos hallazgos y la evaluación traumatológica y ortopédica, se concluyó el diagnóstico de MOT. Se decidió observación y tratamiento conservador. Se controló mensualmente, de forma ambulatoria, evidenciándose disminución progresiva del tamaño de la lesión y, al cuarto mes, no se palpaba la masa tumoral, que no requirió control radiológico; por lo tanto, la paciente fue egresada de la consulta de traumatología y ortopedia.

\section{Discusión}

La MOT está relacionada con un traumatismo evidente y directo de una lesión grave única o episodios recurrentes de traumatismos menores; ${ }^{1}$ en nuestro caso, el desencadenante fue la colocación de un CIVP en la zona lesionada. Se han descrito otros factores de riesgo, como quemaduras graves, sangrado muscular debido a hemofilia, y operaciones ortopédicas; en lactantes, se debe sospechar síndrome del niño maltratado. ${ }^{1}$ Ocurre por una respuesta mesenquimal proliferativa debido a una lesión iniciadora en el tejido blando. En la primera semana, predominan las células fibroblásticas proliferativas y muy vascularizadas. Con la maduración de la lesión, se desarrolla un patrón zonal típico con tres zonas distintas: 1) el centro, que consiste en fibroblastos de proliferación rápida con áreas de hemorragia y músculos necróticos; 2) la zona intermedia, que se caracteriza por osteoblastos con una formación osteoide inmadura e islas de cartílago debido a la osificación endocondral; y 3) la zona periférica, que está compuesta de hueso maduro, generalmente bien separado del tejido circundante por tejido fibroso mixoide. Luego, de la tercera a la cuarta semanas, aparecen calcificaciones y osificaciones dentro de la masa; entre la sexta y la octava semanas, se desarrolla en la periferia un hueso cortical bien organizado, con corteza y espacio de la médula ósea. Este nuevo hueso continúa madurando, de modo que, a los seis meses, se ha desarrollado un anillo denso de hueso compacto con un núcleo central de hueso laminar. ${ }^{8}$

Los mecanismos moleculares de $\mathrm{OH}$ no se han aclarado completamente; sin embargo, una de las moléculas más estudiadas son las PMOs, ${ }^{9}$ que se conoce que inducen la diferenciación de las células fibroblásticas proliferativas en condroblastos y osteoblastos. ${ }^{1}$ La sobreexpresión de PMOs inhibe la alineación y fusión de los mioblastos que se requieren para la formación de los miotubos. Los mioblastos se diferencian en osteoblastos después de la exposición a la señalización de estas proteínas. ${ }^{10}$

Clínicamente, pueden evidenciarse dos a tres semanas después del traumatismo, por edema y formación de masa firme, pétrea, poco dolorosa, y expansiva; ${ }^{6}$ también puede presentar reducción del movimiento articular, y imitar o coexistir con tromboflebitis, celulitis, u osteomielitis. Nuestra paciente no presentó limitación a la flexión ni extensión de la extremidad proximal de la muñeca izquierda; tampoco, signos de infección. ${ }^{4}$

Con respecto a los laboratorios, los nveles de FA pueden ser tan altos como 3.5 veces los niveles normales a las 4 semanas de la lesión, con niveles máximos medidos a las 12 semanas. La elevación de los niveles de FA sérica está acompañada por un aumento en los noveles de fosfato inorgánico, y está precedida por una disminución transitoria de los niveles de calcio sérico. ${ }^{11}$ Una VSG superior a $35 \mathrm{~mm} /$ hora puede indicar el desarrollo de $\mathrm{OH}$. Los niveles de proteína $\mathrm{C}$ reactiva pueden elevarse a principios de la $\mathrm{OH}$, y los niveles de $\mathrm{CQ}$ se pueden usar para determinar la gravedad de la $\mathrm{OH}^{7}$ Ninguno de estos parámetros bioquímicos son específicos, ${ }^{7,11} \mathrm{y}$, en nuestro caso, todos se encontraban dentro de límites normales.

En la Rx simple, las calcificaciones suelen aparecer entre las dos y tres semanas, con un patrón más claro entre las cuatro $\mathrm{y}$ seis semanas postraumatismo. ${ }^{12}$ El sello 
radiográfico más importante es la presencia del llamado fenómeno zonal, que se caracteriza por un área radiolúcida central, que indica una formación ósea inmadura, y por una zona densa periférica de osificación madura. Además, una hendidura radiolúcida delgada separa la masa osificada de la corteza adyacente. ${ }^{8}$ En la mayoría de los casos, la lesión no se adhiere al hueso subyacente, ${ }^{6,8}$ lo que también se cumplió en este caso clínico. En la ecografía se puede apreciar infiltración de la grasa subcutánea y del músculo, y las líneas hiperecogénicas con sombra acústica posterior, aunque estas no aparecen hasta las dos a tres semanas de evolución. ${ }^{6}$ En la resonancia magnética (RM), aparece como una masa de tejido blando no homogénea. En sus primeras etapas, sólo un nódulo isointenso se puede distinguir de los músculos circundantes en imágenes ponderadas en T1, que aumentan el contraste en su periferia en T2 . Sólo en las etapas posteriores los hallazgos de la RM son compatibles con la MO. La tomografía computarizada (TC) generalmente muestra un borde de mineralización alrededor de la lesión después de cuatro a seis semanas; este borde es mucho menos evidente que en la RM. ${ }^{8}$ La TC y la RM poseen mayor resolución que la ecografía, pero no se solicitan de rutina. ${ }^{6}$ La gammagrafía ósea trifásica es más sensible para la detección temprana, aproximadamente 2,5 semanas después de la lesión. ${ }^{4}$ Se podría realizar biopsia si existe duda diagnóstica, o en los casos que no muestran el patrón de osificación zonal radiográfico típico. ${ }^{8}$ Microscópicamente, se observan dos regiones definidas: una zona periférica con osificación, y una zona central celular. En la región exterior, se ve hueso maduro lamelar con osteoclastos activos. En ocasiones, se observa una zona intermedia osteoide, con cartílago o formación de tejido óseo y osteoblastos activos, y una región central, que se compone habitualmente de tejido fibrovascular, que contiene células fusiformes $\mathrm{y}$ células mesénquimales. Esta presentación es patognomónica, y la distingue del osteosarcoma. ${ }^{12}$

En nuestro caso, no se realizó RM, TC, ni la biopsia, porque se contaba con el antecedente de los microtraumas ocasionados por la colocación del CIVP en varias oportunidades en la zona donde se desarrolló la lesión, y con una Rx simple compatible con MOT.

Una entidad importante de diferenciar es la MO progresiva, que está presente en la primera década de vida y se asocia con osificación extraesquelética progresiva y manifestaciones desfigurantes. Este trastorno crónico se reconoce fácilmente por la microdactilia o la adactilia asociadas con el primer dedo del pie y el pulgar. Nuestra paciente no tenía ninguna de estas deformidades. Otros diagnósticos diferenciales serían: hematoma calcificado (depósito de calcio en el músculo), sarcomas de partes blandas, osteosarcoma, hiperparatiroidismo, sarcoidosis, dermatomiositis, y secuela de la necrosis de grasa subcutánea después de la extravasación de gluconato de calcio. $^{4}$

En cuanto al tratamiento, la resolución es espontánea; en la mayoría de los casos, la extirpación quirúrgica de la lesión calcificada no es necesaria en niños, a menos que sea dolorosa o interfiera con el movimiento. ${ }^{1}$ En nuestro caso, se decidió por observación, pues no había limitación a la movilización, no impresionaba dolor a la palpación y, tampoco, progresión del tamaño de la lesión. El seguimiento se realizó hasta el cuarto mes, cuando no se logró palpar la masa tumoral.

\section{Conclusión}

En edad pediátrica y más aún en el RN, es infrecuente observar el desarrollo de MO; este caso resulta interesante, pues se debe a la variedad traumática por el antecedente de la colocación de CIVP, y la lesión fue corroborada con radiología simple. El estudio histológico queda reservado para aquellos pacientes que no presenten un patrón típico de MOT en los estudios imagenológicos. Según la literatura y como sucedió en el presente caso, la MOT sigue un curso benigno, autolimitado, y no requiere cirugía en los niños. Es importante tener en cuenta los diagnósticos diferenciales genéticos y malignos, realizando una historia clínica detallada para tomar una conducta adecuada.

\section{Financiamiento}

Los autores no tienen fuentes de finaciamiento que declarar con relación a este estudio.

\section{Conflicto de Intereses}

Los autores no tienen conflicto de intereses que declarar.

\section{Referencias}

1 Sferopoulos NK, Kotakidou R, Petropoulos AS. Myositis ossificans in children: a review. Eur J Orthop Surg Traumatol 2017;27(04):491-502 https://www.ncbi.nlm.nih.gov/pubmed/ 28275867 cited2019Jul10 [Internet]

2 Samuelson KM, Coleman SS. Nontraumatic myositis ossificans in healthy individuals. JAMA 1976;235(11):1132-1133 https:// jamanetwork.com/journals/jama/article-abstract/344369 cited 2019Jun10 [Internet]

3 Gindele A, Schwamborn D, Tsironis K, Benz-Bohm G. Myositis ossificans traumatica in young children: report of three cases and review of the literature. Pediatr Radiol 2000;30(07):451-459 https://www.ncbi.nlm.nih.gov/pubmed/10929363 cited2019May30 [Internet]

4 Khoo S, Felix L, Azura L, Manmohan S, Jeffry A. A rare case of heterotopic ossification in a newborn: a case report. Malays Orthop J 2012;6(03):48-50 https://www.ncbi.nlm.nih.gov/pmc/ articles/PMC4093592/ cited2019Jun15 [Internet]

5 Simón T, Guillodo Y, Madouas G, Saraux A. Myositis ossificans traumatica (circumscripta) and return to sport: A retrospective series of 19 cases. Joint Bone Spine 2016;83(04):416-420 https:// www.ncbi.nlm.nih.gov/pubmed/26934992 cited2019Jun20 [Internet]

6 Martin R. Miositis osificante traumática: a propósito de un caso. Rev Pediatr Aten Primaria 2015;17:347-350 [citado el 28 de Marzo de 2018] http://scielo.isciii.es/scielo.php?script=sci_ arttext\&pid=S1139-76322015000500011

7 Sun E, Hanyu-Deutmeyer AA. Heterotopic Ossification. [Updated 2019 Jun 4]. In: StatPearls [Internet]. Treasure Island (FL): StatPearls Publishing; 2019 Jan. Available from:https://www. ncbi.nlm.nih.gov/books/NBK519029/

8 Micheli A, Trapani S, Brizzi I, Campanacci D, Resti M, de Martino M. Myositis ossificans circumscripta: a paediatric case and review of the literature. Eur J Pediatr 2009;168(05):523-529 
https://www.ncbi.nlm.nih.gov/pubmed/19130083 cited2019Jun20 [Internet]

9 Kengo S, Kenta U, Takuo K, Masahiro I. The pathophysiology of heterotopic ossification: Current treatment considerations in dentistry. Jpn Dent Sci Rev 2014;50:1-8 Available from https:// www.sciencedirect.com/science/article/pii/S1882761613000549 cited 2019 Jun 27 [Internet]

10 Jitariu A, Heredea R, Ceausu A. Myositis ossificans - a case report and review of literature. Research and Clinical Medicine 2016;1 (01):26-29 [cited 2019 Jun 27] Available from: https://pdfs. semanticscholar.org/a0cf/b42de45dec7ef3ac641232335e533a64b3fa.pdf

11 Nemours: Children's Health System [Internet]. Wilmington: Alfred I. duPont Institute of the Nemours Foundation. Nemours Pediatric Orthopedics; 1996 [cited 2019 Jul 20]. Available from: http://gait.aidi.udel.edu/educate/hobone.htm/

12 Janeiro S, García A, Molina I, Ramos M, Morey M, Iriarte J. Miositis osificante traumática. Rev Esp Cir Oral Maxilofac 2013;35(03): 137-143 [citado el 20 de Mayo del 2019] Disponible en: http:// scielo.isciii.es/pdf/maxi/v35n3/soluciones1.pdf 